\title{
HALL EFFECT ON THERMAL INSTABILITY OF VISCOELASTIC DUSTY FLUID IN POROUS MEDIUM
}

\author{
M. SINGH* \\ Department of Mathematics, Govt Post Graduate College \\ Seema (Rohru) Shimla, INDIA \\ E-mail: Mahinder_singh91@rediffmail.in \\ R.K. GUPTA \\ Department of Mathematics \\ Lovely School of Engineering and Technology \\ Lovely Professional University \\ Phagwara, INDIA
}

\begin{abstract}
The effect of Hall currents and suspended dusty particles on the hydromagnetic stability of a compressible, electrically conducting Rivlin-Ericksen elastico viscous fluid in a porous medium is considered. Following the linearized stability theory and normal mode analysis the dispersion relation is obtained. For the case of stationary convection, Hall currents and suspended particles are found to have destabilizing effects whereas compressibility and magnetic field have stabilizing effects on the system. The medium permeability, however, has stabilizing and destabilizing effects on thermal instability in contrast to its destabilizing effect in the absence of the magnetic field. The critical Rayleigh numbers and the wave numbers of the associated disturbances for the onset of instability as stationary convection are obtained and the behavior of various parameters on critical thermal Rayleigh numbers are depicted graphically. The magnetic field, Hall currents and viscoelasticity parameter are found to introduce oscillatory modes in the systems, which did not exist in the absence of these parameters.
\end{abstract}

Key words: thermal instability, Rivlin-Ericksen viscoelastic fluid, suspended particles, Hall current effect, porous medium.

AMS Classification (2010): 76D50, 76A05, 76A10, 76S05.

\section{Introduction}

The theoretical and experimental results of the onset of thermal instability (Benard convection), under varying assumptions of hydrodynamics and hydromagnetics, have been discussed by Chandrasekhar (1981) in his celebrated monograph. If an electric field is applied at right angles to the magnetic field, the whole current will not flow along the electric field. This tendency of the electric current is called the Hall current effect. The Hall effect is likely to be important in many geophysical and astrophysical situations as well as in flows of laboratory plasma. Sherman and Sutton (1962) considered the effect of Hall currents on the efficiency of a magneto-fluid-dynamic generator. Gupta (1967) studied the problem of thermal instability in the presence of Hall currents and found that Hall currents have a destabilizing effect on the thermal instability of a horizontal layer of a conducting fluid in the presence of a uniform vertical magnetic field. The use of the Boussinesq approximation has been made throughout, which states that the variations of density in the equations of motion can safely be ignored everywhere except in association with the external force. The approximation is well justified in the case of incompressible fluids.

When the fluids are compressible, the equations governing the system become quite complicated. To simplify them, Boussinesq tried to justify the approximation for compressible fluids when the density

\footnotetext{
* To whom correspondence should be addressed
} 
variations arise principally from thermal effects. Spiegel and Veronis' (1960) simplified the set of equations governing the flow of compressible fluids under the following assumptions:

(a) The depth of the fluid layer is much less than the scale height, as defined by them; and (b) The fluctuations in temperature, density and pressure, introduced due to motion, do not exceed their total static variations.

Under the above approximations, the flow equations are the same as those for incompressible fluids, except that the static temperature gradient is replaced by its excess over the adiabatic one and $C_{v}$ is replaced by $C_{p}$.

In geophysical situations, the fluid is often not pure but contains suspended particles. Scanlon and Segel (1973) considered the effects of suspended particles on the onset of Be'nard convection and found that the critical Rayleigh number is reduced because of the heat capacity of the particles. The suspended particles were thus found to destabilize the layer. Palaniswamy and Purushotham (1981) studied the stability of shear flow of stratified fluids with fine dust and found the fine dust to increase the region of instability. The fluids were considered to be Newtonian and the medium was considered to be non-porous in all the above studies.

There is growing importance of non-Newtonian fluids in geophysical fluid dynamics, chemical technology and petroleum industry. Bhatia and Steiner (1972) studied the problem of thermal instability of a Maxwellian viscoelastic fluid in the presence of rotation and found that rotation has a destabilizing influence in contrast to the stabilizing effect on an ordinary viscous (Newtonian) fluid. The thermal instability of an Oldroydian viscoelastic fluid acted on by a uniform rotation was studied by Sharma (1976). There are many elastico-viscous fluids that cannot be characterized by Maxwell's or Oldroyd's constitutive relations. The Rivlin-Ericksen elastico-viscous fluid is one such fluid. Rivlin and Ericksen (1955) studied the stressdeformation relaxations for isotropic materials. Thermal instability in Rivlin-Ericksen elastico-viscous fluids in the presence of rotation and magnetic field, separately, was investigated by Sharma and Kumar (1996; 1997a). Sharma and Kumar (1997b) studied the hydromagnetic stability of two Rivlin-Ericksen elasticoviscous superposed conducting fluids. Kumar and Singh (2006) studied the stability of two superposed Rivlin-Ericksen viscoelastic fluids in the presence of suspended particles. In another study, Kumar et al. (2007) studied the hydrodynamic and hydromagnetic stability of two stratified Rivlin-Ericksen elasticoviscous superposed fluids.

The flow through porous media is of considerable interest for petroleum engineers and geophysical fluid dynamicists. A great number of applications in geophysics may be found in the books by Phillips (1991), Ingham and Pop (1998), and Nield and Bejan (1999). When the fluid slowly percolates through the pores of a macroscopically homogeneous and isotropic porous medium, the gross effect is represented by Darcy's law. As a result of this macroscopic law, the usual viscous term in the equations of fluid motion is replaced by the resistance term $-\frac{1}{k_{l}}\left(\mu+\mu^{\prime} \frac{\partial}{\partial t}\right) \boldsymbol{q}$, where $\mu$ and $\mu^{\prime}$ are the viscosity and viscoelasticity of the Rivlin-Ericksen fluid, $k_{l}$ is the medium permeability and $\boldsymbol{q}$ is the Darcian (filter) velocity of the fluid. Lapwood (1948) studied the stability of a convective flow in hydromagnetics in a porous medium using Rayleigh's procedure. The Rayleigh instability of a thermal boundary layer in flow through a porous medium was considered by Wooding (1960). The stability of superposed Rivlin-Ericksen elastico-viscous fluids permeated with suspended particles in a porous medium was considered by Kumar (2000). Kumar et al. (2004) studied the instability of two rotating viscoelastic (Rivlin-Ericksen) superposed fluids with suspended particles in a porous medium. In another study, Kumar et al. (2005) considered the MHD instability of rotating superposed Rivlin-Ericksen viscoelastic fluids through a porous medium.

Our interest, in the present paper is to bring out the suspended particles effect on thermal instability of a compressible viscoelastic (Rivlin-Ericksen) fluid in a porous medium including the effect of Hall currents. 


\section{Formulation of the problem and perturbation equations}

Consider an infinite horizontal, compressible viscoelastic (Rivlin-Ericksen) fluid-particle layer of thickness $d$ confined between the planes $z=0$ and $z=d$ in the presence of a uniform vertical magnetic field $\boldsymbol{H}(0,0, H)$ in a porous medium. This layer is heated from below such that a steady adverse temperature gradient $\beta(=|d T / d z|)$ is maintained. The equations of motion and continuity for the fluid are

$$
\begin{aligned}
& \frac{\rho}{\varepsilon}\left[\frac{\partial \boldsymbol{v}}{\partial t}+\frac{1}{\varepsilon}(\boldsymbol{v} \cdot \nabla) \boldsymbol{v}\right]=-\nabla p-\rho g \lambda-\frac{1}{k_{l}}\left(\mu+\mu^{\prime} \frac{\partial}{\partial t}\right) \boldsymbol{v}+\frac{K N}{\varepsilon}(\boldsymbol{u}-\boldsymbol{v})+\frac{\mu_{e}}{4 \pi}(\nabla \times \boldsymbol{H}) \times \boldsymbol{H}, \\
& \varepsilon \frac{\partial \rho}{\partial t}+\nabla \cdot(\rho \boldsymbol{v})=0
\end{aligned}
$$

where $\rho, \mu, \mu^{\prime}, p$ and $v(u, v, w)$ denote, respectively, the density, viscosity, viscoelasticity, pressure and the velocity of the pure fluid. Here $\boldsymbol{u}(l, r, s), N(\bar{x}, t)$ denote the velocity and number density of the suspended particles, $\varepsilon$ is medium porosity, $k_{l}$ is medium permeability, $\mu_{e}$ is magnetic permeability, $g$ is acceleration due to gravity, $\bar{x}=(x, y, z), \lambda=(0,0,1)$ and $K=6 \pi \mu \eta^{\prime}, \eta^{\prime}$ being the particle radius, is the Stokes' drag coefficient. Assuming a uniform particle size, spherical shape and small relative velocities between the fluid and particles, the presence of particles adds an extra force term, in the equations of motion Eq.(2.1), proportional to the velocity difference between the particles and the fluid. The force exerted by the fluid on the particles is equal and opposite to that exerted by the particles on the fluid. The buoyancy force on the particles is negligibly small. Interparticles reactions are ignored; we assume that the distances between particles are quite large compared with their diameters. If $m N$ is the mass of particles per unit volume, then the equations of motion and continuity for the particles are

$$
\begin{aligned}
& m N\left[\frac{\partial \boldsymbol{u}}{\partial t}+\frac{1}{\epsilon}(\boldsymbol{u} \cdot \nabla) \boldsymbol{u}\right]=K N(\boldsymbol{v}-\boldsymbol{u}), \\
& \varepsilon \frac{\partial N}{\partial t}+\nabla \cdot(N \boldsymbol{u})=0 .
\end{aligned}
$$

Let $C_{v}, C_{p}, C_{p t}, T$ and $q$ denote, respectively, the heat capacity of the fluid at constant volume, the heat capacity of the fluid at constant pressure, the heat capacity of particles, the temperature and the "effective thermal conductivity" of the pure fluid. Assuming that the fluid particles are in thermal equilibrium, the equation of heat conduction gives

$$
\left[\rho C_{v} \varepsilon+\rho_{s} C_{s}(1-\varepsilon)\right] \frac{\partial T}{\partial t}+\rho C_{v}(\boldsymbol{v} \cdot \nabla) T+m N C_{p t}\left(\varepsilon \frac{\partial}{\partial t}+\boldsymbol{u} \cdot \nabla\right) T=q \nabla^{2} T
$$

where $\rho_{s}, C_{s}$ are the density and the heat capacity of the solid matrix, respectively.

Maxwell's equations in the presence of Hall currents give

$$
\nabla \cdot \boldsymbol{H}=0
$$




$$
\varepsilon \frac{\partial \boldsymbol{H}}{\partial t}=\nabla \times(\boldsymbol{v} \times \boldsymbol{H})+\varepsilon \eta \nabla^{2} \boldsymbol{H}-\frac{c \varepsilon}{4 \pi N^{\prime} e} \nabla \times[(\nabla \times \boldsymbol{H}) \times \boldsymbol{H}]
$$

where $\eta, c, N^{\prime}$ and $e$ denote, respectively, the resistivity, the speed of light, the electron number density and the charge of an electron.

The initial state of the system is taken to be a quiescent layer (no settling) with a uniform particle distribution $N_{O}$ and is given by

$$
\begin{aligned}
& \boldsymbol{v}=(0,0,0), \quad \boldsymbol{H}=(0,0, H), \quad \boldsymbol{u}=(0,0,0), \\
& T=T(z), \quad p=p(z), \quad \rho=\rho(z) \quad \text { and } \quad N=N_{0}=\text { const. }
\end{aligned}
$$

following Spiegel and Veronis' (1960), we have

$$
\begin{aligned}
& T(z)=-\beta z+T_{0}, \quad p(z)=p_{m}-g \int_{0}^{z}\left(\rho_{m}+\rho_{0}\right) d z, \\
& \rho(z)=\rho_{m}\left[1-\alpha_{m}\left(T-T_{m}\right)+K_{m}\left(p-p_{m}\right)\right], \\
& \alpha_{m}=-\left(\frac{1}{\rho} \frac{\partial \rho}{\partial T}\right)_{m}, \quad K_{m}=\left(\frac{1}{\rho} \frac{\partial \rho}{\partial p}\right)_{m} .
\end{aligned}
$$

Spiegel and Veronis' (1960) expressed any state variable say $X$, in the form

$$
X=X_{m}+X_{0}(z)+X^{\prime}(x, y, z, t)
$$

where $X_{m}$ stands for the constant space distribution of $X, X_{0}$ is the variation in $X$ in the absence of motion and $X^{\prime}(x, y, z, t)$ stands for the fluctuations in $X$ due to the motion of the fluid. Also, $\rho_{m}$ and $p_{m}$ stand for the constant space distribution of $\rho$ and $p$ and $\rho_{0}$ and $T_{0}$ stand for the density and temperature of the fluid at the lower boundary. Again following Spiegel and Veronis' (1960) assumptions and results for compressible fluids, the flow equations are found to be the same as those of incompressible fluids except that the static temperature gradient $\beta$ is replaced by its excess over the adiabatic $(\beta-g / C p)$.

Let $\delta p, \delta \rho, \theta, \boldsymbol{v}(u, v, w), \boldsymbol{u}(l, r, s), \boldsymbol{h}\left(h_{x}, h_{y}, h_{z}\right)$ and $N$ denote the perturbations in fluid pressure, density, temperature, velocity, particle velocity, magnetic field $\boldsymbol{H}$ and particle number density $N_{0}$, respectively. Then the linearized hydromagnetic perturbation equations of the viscoelastic fluid-particle layer are

$$
\begin{aligned}
& \frac{1}{\varepsilon} \frac{\partial \boldsymbol{v}}{\partial t}=-\frac{1}{\rho_{m}} \nabla \delta p-g\left(\frac{\delta \rho}{\rho_{m}}\right) \lambda-\frac{1}{k_{l}}\left(v+v^{\prime} \frac{\partial}{\partial t}\right) \boldsymbol{v}+\frac{K N_{0}}{\varepsilon \rho_{m}}(\boldsymbol{u}-\boldsymbol{v})+\frac{\mu_{e}}{4 \pi \rho_{m}}(\nabla \times \boldsymbol{h}) \times \boldsymbol{H}, \\
& \nabla \cdot \boldsymbol{v}=0
\end{aligned}
$$




$$
\begin{aligned}
& m N_{0} \frac{\partial \boldsymbol{u}}{\partial t}=K N_{0}(\boldsymbol{v}-\boldsymbol{u}), \\
& \varepsilon \frac{\partial N}{\partial t}+\nabla \cdot\left(N_{0} \boldsymbol{u}\right)=0, \\
& (E+h \varepsilon) \frac{\partial \theta}{\partial t}=\left(\beta-\frac{g}{C_{p}}\right)(w+h s)+\kappa \nabla^{2} \theta, \\
& \nabla \cdot \boldsymbol{h}=0, \\
& \varepsilon \frac{\partial \boldsymbol{h}}{\partial t}=\nabla \times(\boldsymbol{v} \times \boldsymbol{H})+\varepsilon \eta \nabla^{2} \boldsymbol{h}-\frac{c \varepsilon}{4 \pi N^{\prime} e} \nabla \times[(\nabla \times \boldsymbol{h}) \times \boldsymbol{H}]
\end{aligned}
$$

where $\alpha_{m}=\frac{1}{T_{m}}=\alpha$ (say), $v=\frac{\mu}{\rho_{m}}, \kappa=\frac{q}{\rho_{m} C_{v}}$, and $\frac{g}{C_{p}}, v$ and $\kappa$ stand for the adiabatic gradient, kinematic viscosity and thermal diffusivity, respectively. Also,

$$
h=\frac{f C_{p t}}{C_{v}}, \quad f=\frac{m N_{0}}{\rho_{m}} \quad \text { and } \quad E=\varepsilon+\frac{(1-\varepsilon) \rho_{s} C_{s}}{\rho_{m} C_{v}} .
$$

The linearized dimensionless perturbation equations relevant to the problem are

$$
\begin{aligned}
& N_{p_{1}}^{-1} \frac{\partial u}{\partial t}=-\frac{\partial}{\partial x} \delta p-\frac{1}{P}\left(1+A \frac{\partial}{\partial t}\right) u+\omega(l-u)+N_{Q}\left(\frac{\partial h_{x}}{\partial z}-\frac{\partial h_{z}}{\partial x}\right) \\
& N_{p_{1}}^{-1} \frac{\partial v}{\partial t}=-\frac{\partial}{\partial y} \delta p-\frac{1}{P}\left(1+A \frac{\partial}{\partial t}\right) v+\omega(r-v)+N_{Q}\left(\frac{\partial h_{y}}{\partial z}-\frac{\partial h_{z}}{\partial y}\right), \\
& N_{p_{1}}^{-1} \frac{\partial w}{\partial t}=-\frac{\partial}{\partial z} \delta p-\frac{1}{P}\left(1+A \frac{\partial}{\partial t}\right) w+N_{R} \theta+\omega(s-w), \\
& \frac{\partial u}{\partial x}+\frac{\partial v}{\partial y}+\frac{\partial w}{\partial z}=0, \\
& \left(\tau \frac{\partial}{\partial t}+1\right) l=u, \quad\left(\tau \frac{\partial}{\partial t}+1\right) s=w \\
& \frac{\partial M}{\partial t}+\frac{\partial l}{\partial x}+\frac{\partial r}{\partial y}+\frac{\partial s}{\partial z}=0, \\
& \frac{\partial h_{x}}{\partial x}+\frac{\partial h_{y}}{\partial y}+\frac{\partial h_{z}}{\partial z}=0
\end{aligned}
$$




$$
\begin{aligned}
& N_{p_{2}} N_{p_{1}}^{-1} \frac{\partial h_{x}}{\partial t}=\varepsilon^{-1} \frac{\partial u}{\partial z}+\nabla^{2} h_{x}-M_{1} \frac{\partial}{\partial z}\left(\frac{\partial h_{z}}{\partial y}-\frac{\partial h_{y}}{\partial z}\right), \\
& N_{p_{2}} N_{p_{1}}^{-1} \frac{\partial h_{y}}{\partial t}=\varepsilon^{-1} \frac{\partial v}{\partial z}+\nabla^{2} h_{y}-M_{1} \frac{\partial}{\partial z}\left(\frac{\partial h_{x}}{\partial z}-\frac{\partial h_{z}}{\partial x}\right), \\
& N_{p_{2}} N_{p_{1}}^{-1} \frac{\partial h_{z}}{\partial t}=\varepsilon^{-1} \frac{\partial w}{\partial z}+\nabla^{2} h_{z}-M_{1} \frac{\partial}{\partial z}\left(\frac{\partial h_{y}}{\partial x}-\frac{\partial h_{x}}{\partial y}\right), \\
& (E+h \varepsilon) \frac{\partial \theta}{\partial t}=\left(\frac{G-1}{G}\right)(w+h s)+\nabla^{2} \theta
\end{aligned}
$$

where $N_{p_{1}}=\frac{\varepsilon v}{\kappa}$ is the modified Prandtl number, $N_{p_{2}}=\frac{\varepsilon v}{\eta}$ is the modified magnetic Prandtl number, $N_{R}=\frac{g \alpha \beta d^{4}}{\nu \kappa}$ is the Rayleigh number, $N_{Q}=\frac{\mu_{e} H^{2} d^{2}}{4 \pi \rho_{m} \nu \eta}$ is the Chandrasekhar number, $M=\frac{\varepsilon N}{N_{0}}, \quad M_{1}=\frac{c H}{4 \pi N^{\prime} e \eta}$ is the Hall parameter, $\omega=\frac{K N_{0} d^{2}}{\rho_{m} v \varepsilon}, \tau=\frac{m \kappa}{K d^{2}}, A=\left(\frac{v}{v^{\prime}}\right) \frac{\kappa}{d^{2}}, f=\frac{m N_{0}}{\rho_{m}}=\tau \omega N_{p_{l}}$ is the mass fraction, $G=\frac{C_{p} \beta}{g}$ and $P=\frac{k_{1}}{d^{2}}$. Here physical variables have been scaled using $d, \frac{d^{2}}{\kappa}, \frac{\kappa}{d}, \frac{\rho v \kappa}{d^{2}}, \beta d$ and $\frac{H \kappa}{\eta}$ as the length, time, velocity, pressure, temperature and magnetic field scale factors, respectively.

Now we consider the case of two free boundaries and the medium adjoining the fluid as nonconducting. The boundary conditions appropriate for the problem are

$$
w=\frac{\partial^{2} w}{\partial z^{2}}=\theta=0, \quad \xi=\frac{\partial \zeta}{\partial z}=0, \quad \text { at } \quad z=0 \quad \text { and } \quad z=1,
$$

and

$$
h_{x}, h_{y}, h_{z} \text { are continuous with an external vacuum field. }
$$

Here $\zeta=\left(\frac{\partial v}{\partial x}-\frac{\partial u}{\partial y}\right)$ and $\xi=\left(\frac{\partial h_{y}}{\partial x}-\frac{\partial h_{x}}{\partial y}\right)$ are the $z$-components of vorticity and current density, respectively.

Equations (2.18)-(2.28), after eliminating $u, v$ and $\delta p$, can be expressed as

$$
\left[L_{1}+\frac{L_{2}}{P}\left(1+A \frac{\partial}{\partial t}\right)\right] \nabla^{2} w=L_{2} N_{Q} \nabla^{2} \frac{\partial h_{z}}{\partial z}+L_{2} N_{R} \nabla_{1}^{2} \theta
$$




$$
\begin{aligned}
& {\left[L_{1}+\frac{L_{2}}{P}\left(1+A \frac{\partial}{\partial t}\right)\right] \zeta=L_{2} N_{Q} \frac{\partial \xi}{\partial z},} \\
& {\left[N_{p_{2}} N_{p_{1}}^{-1} \frac{\partial}{\partial t}-\nabla^{2}\right] \xi=\epsilon^{-1} \frac{\partial \zeta}{\partial z}+M_{1} \frac{\partial}{\partial z}\left(\nabla^{2} h_{z}\right),} \\
& {\left[N_{p_{2}} N_{p_{1}}{ }^{-1} \frac{\partial}{\partial t}-\nabla^{2}\right] h_{z}=\epsilon^{-1} \frac{\partial w}{\partial z}-M_{1} \frac{\partial \xi}{\partial z},} \\
& L_{2}\left[(E+h \in) \frac{\partial}{\partial t}-\nabla^{2}\right] \theta=\left(\frac{G-1}{G}\right)\left[\tau \frac{\partial}{\partial t}+\bar{H}\right] w
\end{aligned}
$$

where

$$
\begin{aligned}
& L_{l}=N_{p_{l}}{ }^{-1}\left(\tau \frac{\partial^{2}}{\partial t^{2}}+F \frac{\partial}{\partial t}\right), \quad F=f+1, \quad L_{2}=\tau \frac{\partial}{\partial t}+1, \quad \nabla^{2}=\frac{\partial^{2}}{\partial x^{2}}+\frac{\partial^{2}}{\partial y^{2}}+\frac{\partial^{2}}{\partial z^{2}}, \\
& \nabla_{l}^{2}=\frac{\partial^{2}}{\partial x^{2}}+\frac{\partial^{2}}{\partial y^{2}}, \quad \bar{H}=h+1 .
\end{aligned}
$$

\section{The dispersion relation}

Analyzing the disturbances in terms of normal modes by seeking solutions whose dependence on $x, y$ and $t$ is given by

$$
\left[w, \theta, h_{z}, \zeta, \xi\right]=[W(z), \Theta(z), K(z), Z(z), X(z)] \exp \left(i k_{x} x+i k_{y} y+n t\right)
$$

where $k_{x}$ and $k_{y}$ are the wave numbers along $x$ - and $y$-directions and the resultant wave number is given by $k=\left(k_{x}^{2}+k_{y}^{2}\right)^{1 / 2}$ and $n$ is the growth rate, Eqs (2.31)-(2.35), with the help of expression (3.1) become

$$
\begin{aligned}
& {\left[L_{1}+\frac{L_{2}}{P}\{1+A n\}\right]\left(D^{2}-\alpha^{2}\right) W=L_{2} N_{Q}\left(D^{2}-\alpha^{2}\right) D K-L_{2} N_{R} \alpha^{2} \Theta,} \\
& {\left[L_{1}+\frac{L_{2}}{P}\{1+A n\}\right] Z=L_{2} N_{Q} D X,} \\
& {\left[N_{p_{2}} N_{p_{1}}{ }^{-1} n-\left(D^{2}-\alpha^{2}\right)\right] X=\epsilon^{-1} D Z+M_{1}\left(D^{2}-\alpha^{2}\right) D K,} \\
& {\left[N_{p_{2}} N_{p_{1}}{ }^{-1} n-\left(D^{2}-\alpha^{2}\right)\right] K=\epsilon^{-1} D W-M_{1} D X,}
\end{aligned}
$$




$$
L_{2}\left[(E+h \in) n-\left(D^{2}-\alpha^{2}\right)\right] \Theta=\left(\frac{G-1}{G}\right)[\tau n+\bar{H}] W
$$

where

$$
D=\frac{d}{d z}, \quad L_{1}=N_{p_{1}}^{-1}\left(\tau n^{2}+F n\right) \quad \text { and } \quad L_{2}=\tau n+1 .
$$

Eliminating $Z, X, K$ and $\Theta$ between Eqs (3.2)-(3.6), we obtain

$$
\begin{aligned}
& {\left[L_{1}+\frac{L_{2}}{P}(1+A n)\right]\left[\left(D^{2}-\alpha^{2}\right)-(E+h \varepsilon)\right]\left(D^{2}-\alpha^{2}\right) W+L_{2} N_{Q}\left[\left(D^{2}-\alpha^{2}\right)-(E+h \varepsilon) n\right]} \\
& {\left[\left\{\left(D^{2}-\alpha^{2}\right)-N_{p_{2}} N_{p_{1}}{ }^{-1} n\right\} M_{1}^{-1} \varepsilon^{-1}+L_{2} N_{Q}\left[L_{1}+\frac{L_{2}}{P}(1+A n)\right]^{-1} M_{1}^{-1} \varepsilon^{-2} D^{2}\right] D^{2}} \\
& {\left[M_{1}\left(D^{2}-\alpha^{2}\right) D^{2}+M_{1}^{-1}\left\{\left(D^{2}-\alpha^{2}\right)-N_{p_{2}} N_{p_{1}}{ }^{-1} n\right\}^{2}+L_{2} N_{Q}\left[L_{1}+\frac{L_{2}}{P}(1+A n)\right]^{-1}\right.} \\
& \left.\varepsilon^{-1} M_{1}^{-1}\left\{\left(D^{2}-\alpha^{2}\right)-N_{p_{2}} N_{p_{1}}{ }^{-1} n\right\} D^{2}\right]^{-1}\left(D^{2}-\alpha^{2}\right) W=\left(\frac{G-1}{G}\right) N_{R} \alpha^{2}(\tau n+\bar{H}) .
\end{aligned}
$$

Using the boundary conditions Eqs (2.29) and (2.30), it can be shown that all the even order derivatives of $W$ vanish at the boundaries. Hence the proper solution of Eq.(3.7) characterizing the lowest mode is

$$
W=W_{0} \sin \pi z, \quad \text { where } \quad W_{0} \quad \text { is constant. }
$$

On substituting the solution (3.8) in Eq.(3.7), we get the dispersion relation as

$$
\begin{aligned}
& N_{R}=\left(\frac{G}{G-1}\right) \frac{\left(\pi^{2}+\alpha^{2}\right)\left[\left(\pi^{2}+\alpha^{2}\right)+(E+h \varepsilon) n\right]}{\alpha^{2}(\tau n+\bar{H})}\left[\left(L_{1}+\frac{L_{2}}{P}\{1+A n\}\right)\right]+ \\
& +L_{2} N_{Q} \pi^{2}\left[\left[\left\{\left(\pi^{2}+\alpha^{2}\right)+N_{p_{2}} N_{p_{1}}{ }^{-1} n\right\} M_{1}^{-1} \varepsilon^{-1}+L_{2} N_{Q}\left(L_{1}+\frac{L_{2}}{P}\{1+A n\}\right)^{-1} M_{1}^{-1} \varepsilon^{-2} \pi^{2}\right]\right. \\
& {\left[M_{1} \pi^{2}\left(\pi^{2}+\alpha^{2}\right)+M_{1}^{-1}\left\{\left(\pi^{2}+\alpha^{2}\right)+N_{p_{2}} N_{p_{1}}{ }^{-1} n\right\}^{-2}+L_{2} N_{Q}\left(L_{1}+\frac{L_{2}}{P}(1+A n)\right)^{-1}\right.} \\
& \left.\left.\varepsilon^{-1} M_{1}^{-1}\left\{\left(\pi^{2}+\alpha^{2}\right)+N_{p_{2}} N_{p_{1}}{ }^{-1} n\right\} n\right]^{-1}\right] .
\end{aligned}
$$

\section{Stationary convection}

When the instability sets in as stationary convection, the marginal state will be characterized by $n=$ 0 and the dispersion relation Eq.(3.9) reduces to 


$$
N_{R}=\left(\frac{G}{G-1}\right) \frac{\left(\pi^{2}+\alpha^{2}\right)^{2}}{\alpha^{2} \bar{H}}\left[\frac{1}{P}+\frac{N_{Q} \pi^{2} \varepsilon^{-1}\left\{\left(\pi^{2}+\alpha^{2}\right)+N_{Q} P \varepsilon^{-1} \pi^{2}\right\}}{\left(\pi^{2}+\alpha^{2}\right)\left\{M_{1}^{2} \pi^{2}+\left(\pi^{2}+\alpha^{2}\right)+N_{Q} P \varepsilon^{-1} \pi^{2}\right\}}\right] .
$$

Thus for stationary convection, the viscoelastic parameter $A$ vanishes with $n$, and the RivlinEricksen viscoelastic fluid behaves like a Newtonian fluid. Also, for fixed values of $P, N_{Q}, M_{1}$ and $\bar{H}$, let the non-dimensional number $G$ accounting for the compressibility effects be also kept as fixed, then we have

$$
\overline{N_{R}{ }^{C}}=\left(\frac{G}{G-1}\right) N_{R}{ }^{C}
$$

where $N_{R}{ }^{C}$ and $\overline{N_{R}{ }^{C}}$ denote, respectively, the critical Rayleigh numbers in the absence and presence of compressibility. Since the critical Rayleigh number is positive and finite, so $G>1$ and we obtain a stabilizing effect of compressibility, i.e. its effect is to postpone the onset of thermal convection in fluidparticle layer.

To study the effects of suspended particles, medium permeability, magnetic field and Hall currents, we examine the natures of $\frac{d N_{R}}{d \bar{H}}, \frac{d N_{R}}{d P}, \frac{d N_{R}}{d N_{Q}}$ and $\frac{d N_{R}}{d M_{1}}$, respectively.

From Eq.(4.1), we have

$$
\frac{d N_{R}}{d \bar{H}}=-\left(\frac{G}{G-1}\right) \frac{\left(\pi^{2}+\alpha^{2}\right)^{2}}{\alpha^{2} \bar{H}^{2}}\left[\frac{1}{P}+\frac{N_{Q} \pi^{2} \varepsilon^{-1}\left\{\left(\pi^{2}+\alpha^{2}\right)+N_{Q} P \varepsilon^{-1} \pi^{2}\right\}}{\left(\pi^{2}+\alpha^{2}\right)\left\{M_{1}^{2} \pi^{2}+\left(\pi^{2}+\alpha^{2}\right)+N_{Q} P \varepsilon^{-1} \pi^{2}\right\}}\right],
$$

which is negative. The effect of suspended particles is, thus, destabilizing the thermal instability of the compressible fluid-particle layer in the presence of Hall currents through a porous medium.

It is evident from Eq.(4.1) that

$$
\frac{d N_{R}}{d P}=\left(\frac{G}{G-1}\right) \frac{\left(\pi^{2}+\alpha^{2}\right)^{2}}{\alpha^{2} \bar{H}}\left[-\frac{1}{P^{2}}+\frac{\left(N_{Q} \pi^{2} \varepsilon^{-1}\right) M_{1}^{2} \pi^{2}}{\left(\pi^{2}+\alpha^{2}\right)\left\{M_{l}^{2} \pi^{2}+\left(\pi^{2}+\alpha^{2}\right)+N_{Q} P \varepsilon^{-1} \pi^{2}\right\}^{2}}\right],
$$

which is positive if $P\left[M_{1} \pi-\left(\pi^{2}+\alpha^{2}\right)^{\frac{1}{2}}\right]>\frac{\left(\pi^{2}+\alpha^{2}\right)^{\frac{1}{2}}\left[M_{1}^{2} \pi^{2}+\left(\pi^{2}+\alpha^{2}\right)\right]}{N_{Q} \varepsilon^{-1} \pi^{2}}$,

and is negative if $P\left[M_{1} \pi-\left(\pi^{2}+\alpha^{2}\right)^{\frac{1}{2}}\right]<\frac{\left(\pi^{2}+\alpha^{2}\right)^{\frac{1}{2}}\left[M_{1}^{2} \pi^{2}+\left(\pi^{2}+\alpha^{2}\right)\right]}{N_{Q} \varepsilon^{-1} \pi^{2}}$. 
Thus, the medium permeability has both a stabilizing and destabilizing effect depending upon the values of various parameters. In the absence of the magnetic field, the medium permeability has a destabilizing effect, since for the case

$$
\frac{d N_{R}}{d P}=-\left(\frac{G}{G-1}\right) \frac{\left(\pi^{2}+\alpha^{2}\right)^{2}}{\alpha^{2} \bar{H} P^{2}}
$$

which is always negative. The medium permeability, thus, succeeds in stabilizing the thermal instability of the compressible fluid-particle layer for certain wave numbers in the presence of the magnetic field, which was unstable in the absence of the magnetic field.

Now from Eq.(4.1), we get

$$
\begin{aligned}
& \frac{d N_{R}}{d N_{Q}}=\left(\frac{G}{G-1}\right) \frac{\left(\pi^{2}+\alpha^{2}\right) \pi^{2} \varepsilon^{-1}}{\alpha^{2} \bar{H}}\left[M_{1}^{2} \pi^{2}+\left(\pi^{2}+\alpha^{2}\right)+N_{Q} P \varepsilon^{-1} \pi^{2}\right]^{-1} \\
& {\left[\left\{\left(\pi^{2}+\alpha^{2}\right)+N_{Q} P \varepsilon^{-1} \pi^{2}\right\}+M_{1}^{2} \pi^{4} N_{Q} P \varepsilon^{-1}\left\{M_{1}^{2} \pi^{2}+\left(\pi^{2}+\alpha^{2}\right)+N_{Q} P \varepsilon^{-1} \pi^{2}\right\}^{-1}\right],}
\end{aligned}
$$

which is always positive. This shows that the magnetic field has a stabilizing effect on the system. To find the effect of Hall currents, from Eq.(4.1), we have

$$
\frac{d N_{R}}{d M_{1}}=-2\left(\frac{G}{G-1}\right) \frac{\left(\pi^{2}+\alpha^{2}\right) N_{Q} \varepsilon^{-1} M_{1} \pi^{4}\left\{\left(\pi^{2}+\alpha^{2}\right)+N_{Q} P \varepsilon^{-1} \pi^{2}\right\}}{\alpha^{2} \bar{H}\left\{M_{1}^{2} \pi^{2}+\left(\pi^{2}+\alpha^{2}\right)+N_{Q} P \varepsilon^{-1} \pi^{2}\right\}^{2}},
$$

which is always negative. Therefore, the effect of Hall currents is destabilizing on the thermal convection in the compressible fluid-particle layer in a porous medium.

When we analyze graphically all the four effects, we find from Fig.1, when the value of the medium permeability $(P)$, increased then the value of $N_{R}$ is increased. From Fig.2, similarly to the medium permeability, the value of the magnetic field $\left(N_{Q}\right)$ increased, and the value of $N_{R}$ is increased, showing the case of stabilizing effect. In Fig.3, as the value of suspended particle $(\bar{H})$, increased, the value of $N_{R}$ decreased in the presence of Hall currents through the porous medium, showing the case of destabilizing effect on the system. 


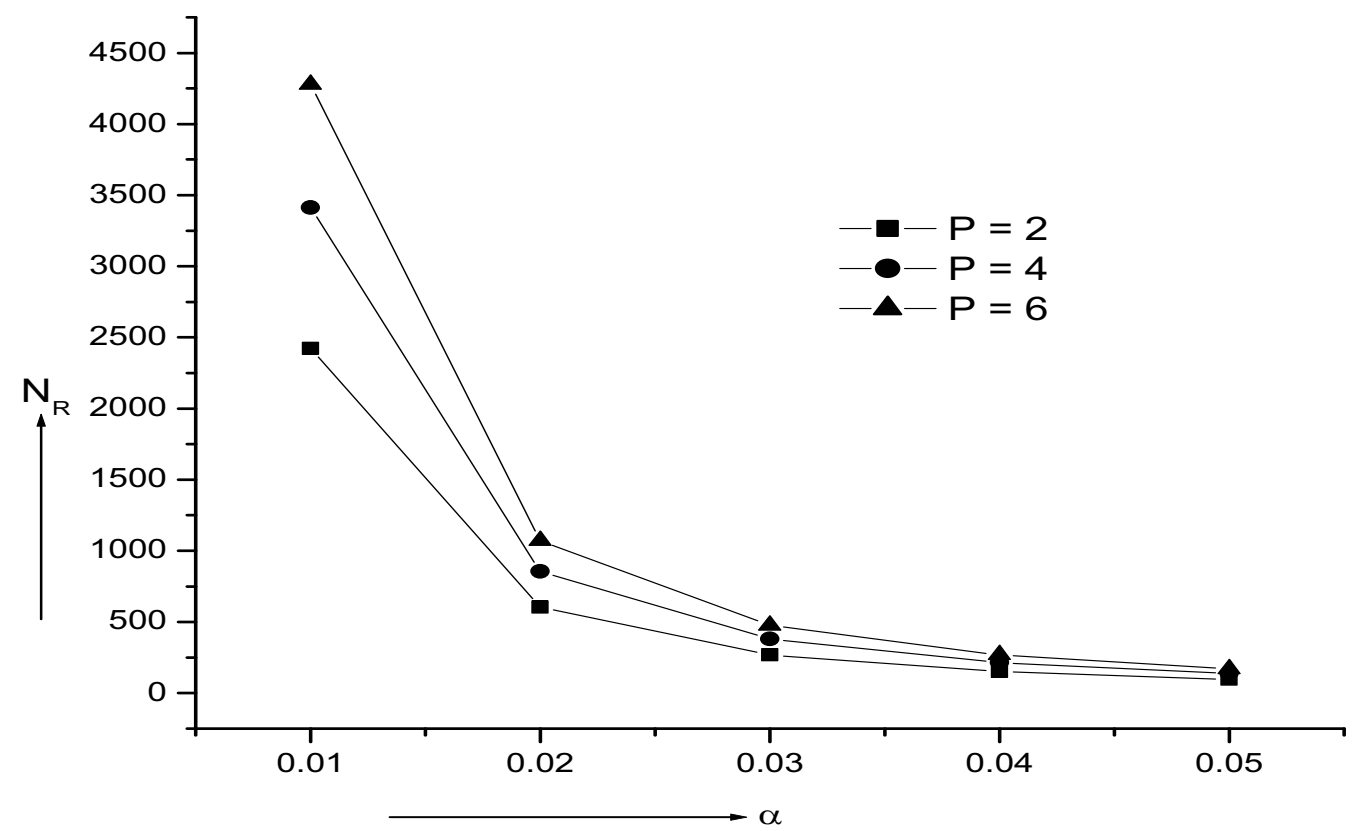

Fig.1. Variation of $N_{R}$ with $\alpha$ for a fixed $\bar{H}=1000, G=9.8, \pi=3.14, N_{Q}=20, \mathrm{M}_{1}=10, \varepsilon=0.5$ and for different values of $P(2,4,0)$.

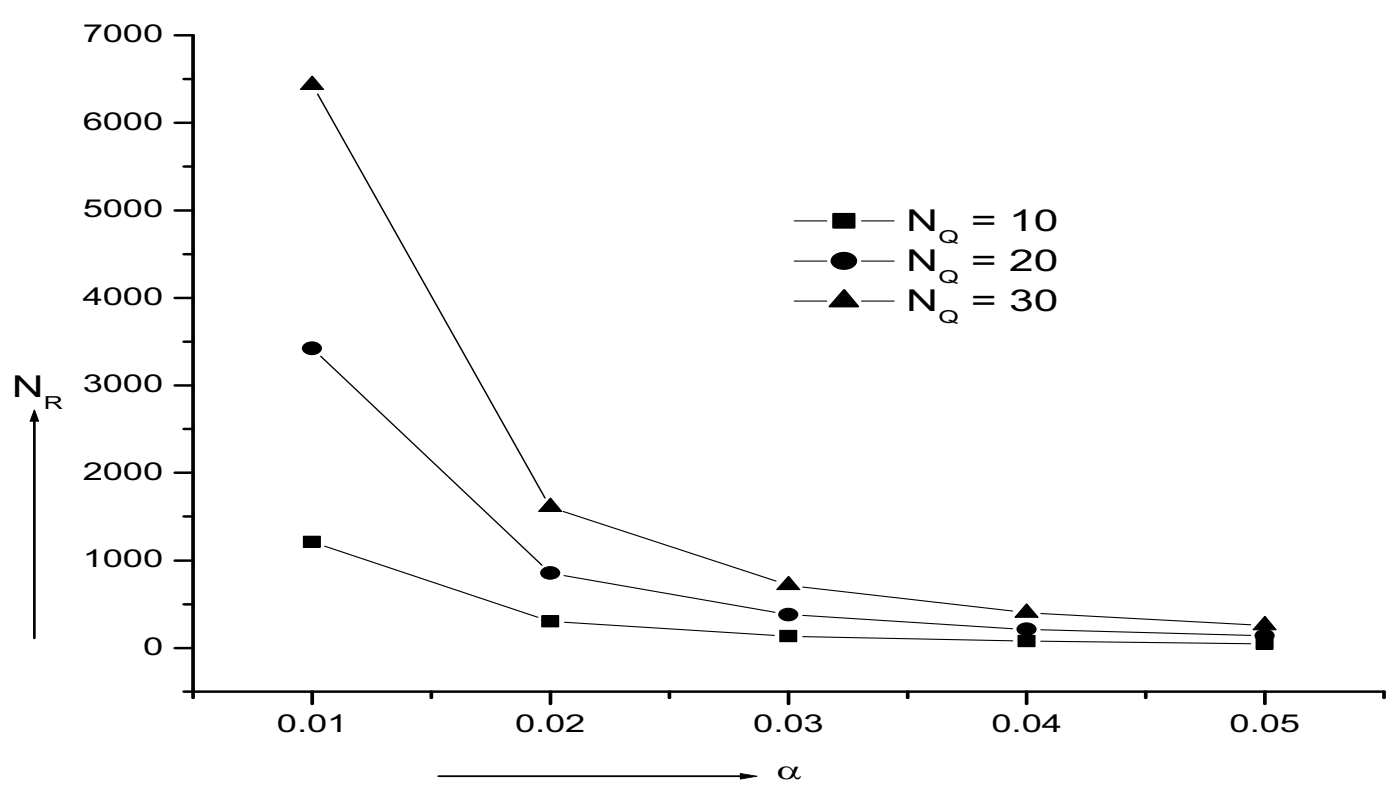

Fig.2. Variation of $N_{R}$ with $\alpha$ for a fixed $\bar{H}=1000, G=9.8, \pi=3.14, P=4, \mathrm{M}_{1}=10, \varepsilon=0.5$ and for different values of $N_{Q}(10,20,30)$. 


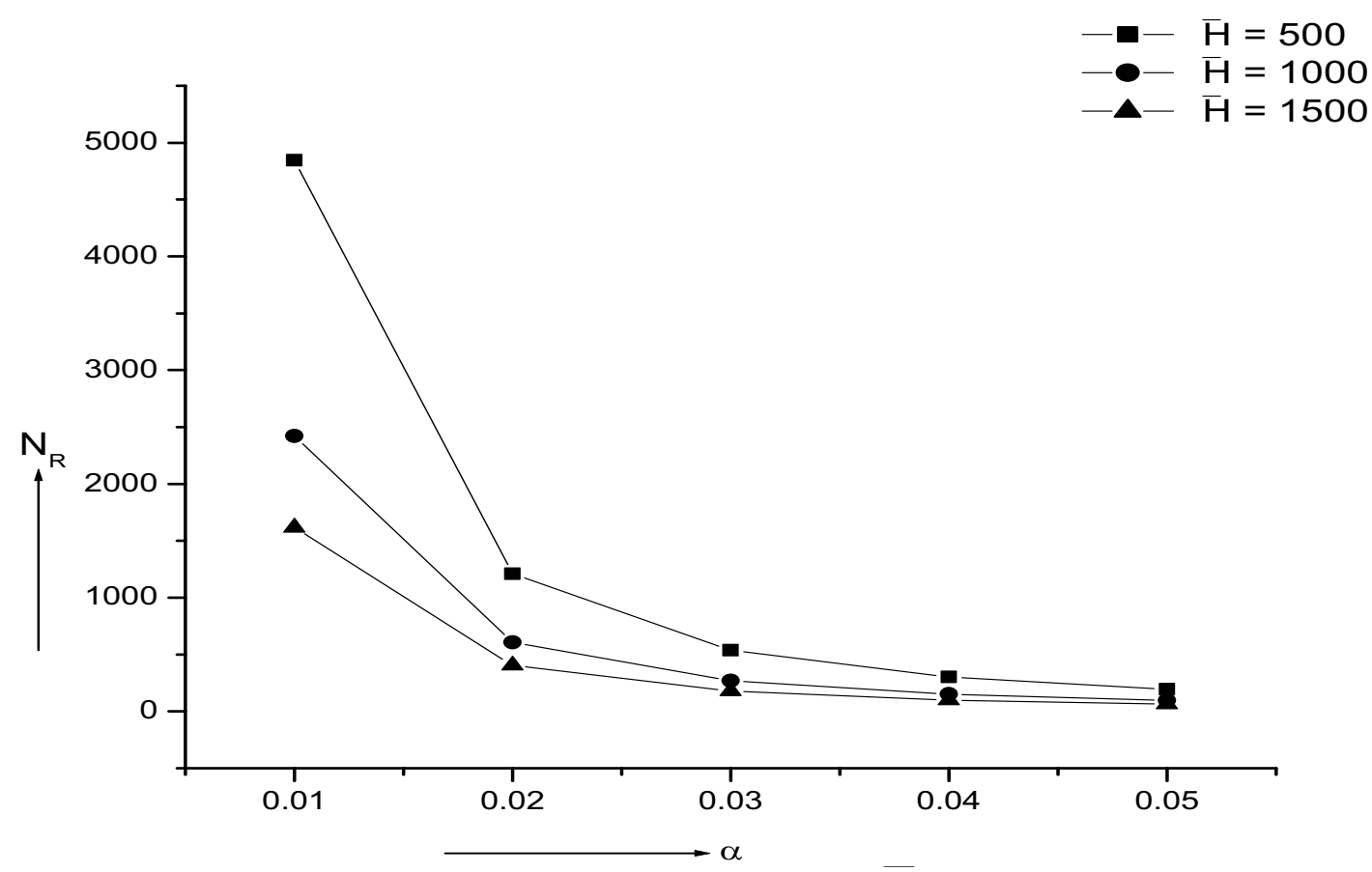

Fig.3. Variation of $N_{R}$ with $\alpha$ for a different value of $\bar{H}(=500,1000,1500)$ for fixed values of $G=9.8, P=2, \pi=3.14, N_{Q}=20, \mathrm{M}_{1}=10, \varepsilon=0.5$.

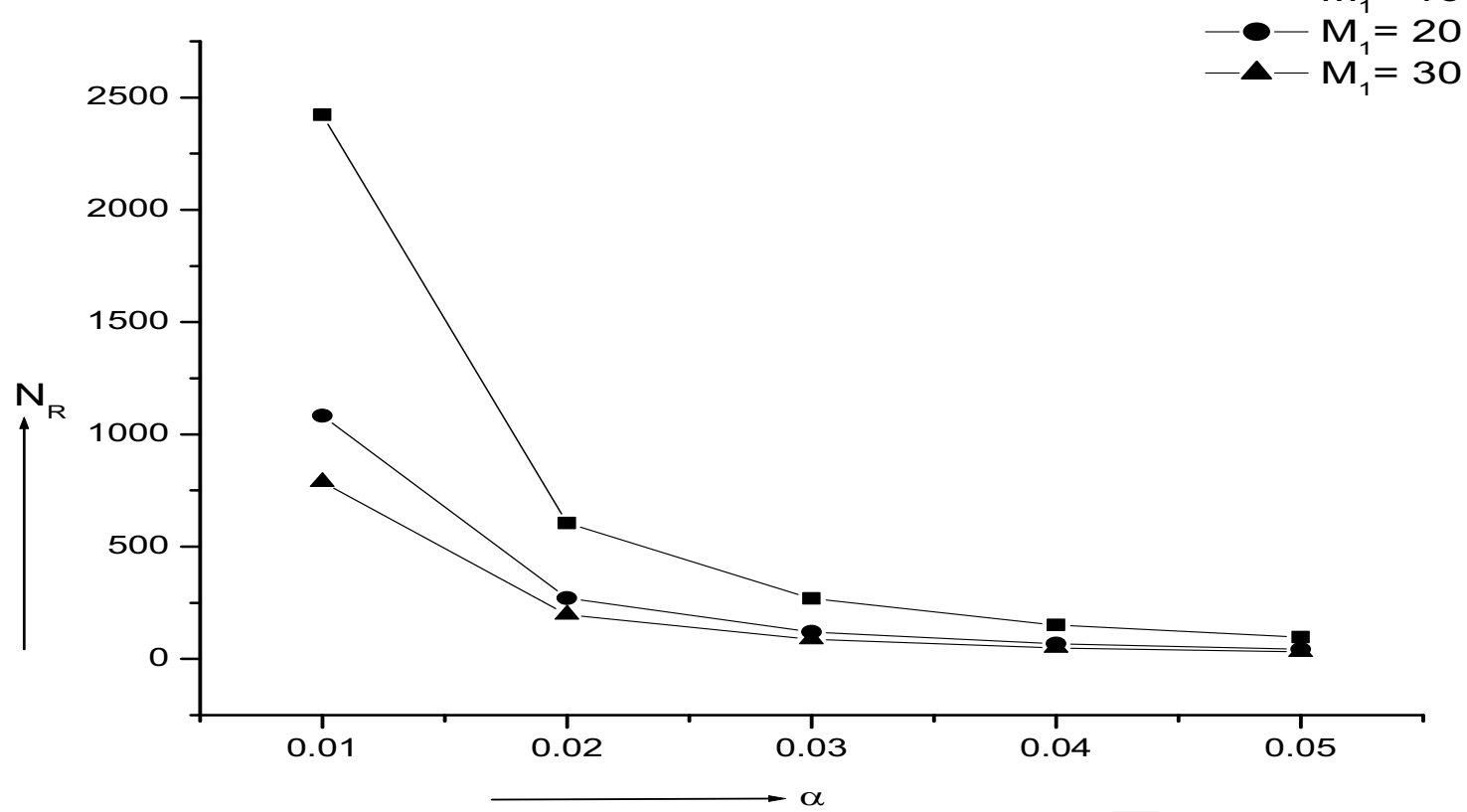

Fig.4. Variation of $N_{R}$ with $\alpha$ for a fixed values $\bar{H}=1000, G=9.8, \pi=3.14, P=2, N \Theta=20, \varepsilon=0.5$, for different value of $\mathrm{M}_{1}=(10,20,30)$. 


\section{Existence of oscillatory modes}

Multiplying Eq.(3.2) by $W^{*}$, the complex conjugate of $W$, integrating over the range of $z$ and using Eqs (3.3)-(4.3) together with the boundary conditions (2.29) and (2.30), we obtain

$$
\begin{array}{ll}
{\left[L_{1}+\frac{L_{2}}{P}(1+A n)\right] I_{1}+L_{2} N_{Q} \varepsilon N_{p_{2}} N_{p_{1}}{ }^{-1}\left(n I_{2}+n^{*} I_{5}\right)+L_{2} N_{Q} \varepsilon\left(I_{3}+I_{6}\right)+} \\
+\frac{L_{2}}{L_{2}^{*}}\left[L_{1}^{*}+\frac{L_{2}^{*}}{P} l+\left(A n^{*}\right)\right] I_{4}=L_{2} L_{2}{ }_{2} N_{R} \alpha^{2}\left(\frac{G}{G-1}\right)\left(\frac{1}{\tau n^{*}+\bar{H}}\right)\left\{I_{7}+(E+h \in) n^{*} I_{8}\right\} \\
I_{1}=\int_{0}^{1}\left(|D W|^{2}+\alpha^{2}|W|^{2}\right) d z, & I_{2}=\int_{0}^{1}|X|^{2} d z, \\
I_{3}=\int_{0}^{1}\left(|D X|^{2}+\alpha^{2}|X|^{2}\right) d z, & I_{4}=\int_{0}^{1}|Z|^{2} d z, \\
I_{5}=\int_{0}^{1}\left(|D K|^{2}+\alpha^{2}|K|^{2}\right) d z, & I_{6}=\int_{0}^{1}\left(\left|D^{2} K\right|^{2}+2 \alpha^{2}|D K|^{2}+\alpha^{4}|K|^{2}\right) d z \\
I_{7}=\int_{0}^{1}\left(|D \Theta|^{2}+\alpha^{2}|\Theta|^{2}\right) d z, & I_{8}=\int_{0}^{1}|\Theta|^{2} d z,
\end{array}
$$

which all are positive definite. Putting $n=i n_{0}$, where $n_{0}$ is real, in Eq.(5.1) and equating imaginary parts on both sides, we obtain either

$$
n_{0}=0
$$

or

$$
\begin{aligned}
& n_{0}{ }^{2}=-\tau^{-2}\left[\left(N_{p_{1}}{ }^{-1} \bar{H} F-\frac{\tau}{P}-\frac{\tau}{P} A\right) I_{1}+\right. \\
& +N_{Q} \varepsilon N_{p_{2}} N_{p_{1}}{ }^{-1} \bar{H}\left(I_{2}-I_{5}\right)-N_{Q} \varepsilon \tau\left(I_{3}+I_{6}\right)-\left(N_{p_{1}}{ }^{-1} \bar{H} F+\frac{\tau}{P}+\frac{\tau}{P} A\right) I_{4}+ \\
& \left.+N_{R} \alpha^{2}\left(\frac{G}{G-1}\right)\left\{\tau I_{7}+(E+h \varepsilon) I_{8}\right\}\right]\left[\left\{N_{p_{1}}^{-1}(\bar{H}+1-F)-\frac{\tau}{P}-\frac{\tau}{P} A\right\} I_{1}+\right. \\
& +N_{Q^{\varepsilon}} \varepsilon N_{p_{2}} N_{p_{1}}{ }^{-1} \bar{H}\left(I_{2}-I_{5}\right)-N_{Q} \varepsilon \tau\left(I_{3}+I_{6}\right)+\left\{N_{p_{1}}{ }^{-1}(1-\bar{H}-F)-\frac{\tau}{P}-\frac{\tau}{P} A\right\} I_{4}+ \\
& \left.+N_{R} \alpha^{2}\left(\frac{G}{G-1}\right)\left\{\tau I_{7}+(E+h \varepsilon) I_{8}\right\}\right]^{-1} .
\end{aligned}
$$

In the absence of the magnetic field 


$$
n_{0}^{2}=\frac{\left[\left(N_{p_{1}}{ }^{-1} \bar{H} F-\frac{\tau}{P}-\frac{\tau}{P} A\right) I_{1}+N_{R} \alpha^{2}\left(\frac{G}{G-1}\right)\left\{\tau I_{7}+(E+h \varepsilon) I_{8}\right\}\right]}{\tau^{2}\left[\left\{N_{p_{1}}{ }^{-1}(\bar{H}+1-F)-\frac{\tau}{P}-\frac{\tau}{P} A\right\} I_{1}+N_{R} \alpha^{2}\left(\frac{G}{G-1}\right)\left\{\tau I_{7}+(E+h \varepsilon) I_{8}\right\}\right]} .
$$

\section{Conclusion}

The combined effect of various parameters that is the magnetic field, compressibility, medium permeability, Hall currents and suspended particles on thermal instability of a Rivlin-Ericksen fluid has been investigated. The principal conclusions are the following.

(I) The stationary convection Rivlin-Ericksen fluid behaves like an ordinary Newtonian fluid due to the vanishing of the viscoelastic parameter.

(II) The presence of the magnetic field (and therefore Hall currents), suspended particles and medium permeability effects introduce oscillatory modes in the system, in the absence of these effects the principle of exchange of stabilities is found to hold good.

(III) In the absence of the magnetic field, the expression for $n_{0}{ }^{2}$ given by Eq.(5.5), is negative if

$$
C_{p t}>C_{v}\left[1+\frac{\varepsilon m}{f k_{l} K d^{2}}\left\{v d^{2}+v^{\prime} k\right\}\right]
$$

for all positive $N_{R}$, since $n_{0}$ is real and $n_{0}^{2}$ is negative, therefore, we must have $n_{0}=0$. This shows that $n$ is real for positive $N_{R}$ in the absence of the magnetic field if Eq.(5.6) holds true and that the principle of exchange of stabilities is valid for this case, however, if Eq.(5.6) is violated, then the oscillatory modes may come into play even in the absence of the magnetic field.

(IV) From Eq.(4.2), it is clear that the effect of compressibility is to postpone the onset of instability.

(V) To investigate the effects of suspended particles, medium permeability, magnetic filed, and Hall currents in a compressible Rivlin-Ericksen viscoelastic fluid, we examined the expressions $d N_{R} / d \bar{H}, d N_{R} / d P, d N_{R} / d N_{Q}$ and $d N_{R} / d M_{1}$ analytically. The magnetic field postpones the onset of instability, suspended particles and Hall currents both hasten the onset of convection, which is in contrast with the result of Gupta et al. (2012).

\section{Nomenclature}

$$
\begin{aligned}
e_{i j} & - \text { rate of strain tensor } \\
\boldsymbol{g}(0,0,-g) & - \text { gravity force } \\
\boldsymbol{H}(0,0, H) & - \text { magnetic field } \\
h\left(h_{x}, h_{y}, h_{z}\right) & - \text { perturbation in magnetic field } \\
k=\left(k_{x}{ }^{2}+k_{y}{ }^{2}\right)^{1 / 2} & - \text { resultant wave number } \\
k_{l} & - \text { permeability of the medium } \\
M=\left(\frac{c H}{4 \pi N e \eta}\right)^{2} & - \text { non-dimensional number according to the Hall currents } \\
P & - \text { pressure } \\
\mathrm{R}=\frac{g \alpha \beta d^{4}}{v \chi} & - \text { thermal Rayleigh number }
\end{aligned}
$$




$$
\begin{aligned}
S=\frac{g \alpha^{\prime} \beta^{\prime} d^{4}}{v \chi^{\prime}} & - \text { analogous solute Rayleigh number } \\
T & - \text { temperature } \\
\mathrm{Q}=\frac{\mu_{e} H^{2} d^{2}}{4 \pi \rho_{0} v \eta} & - \text { Chandrasekhar number } \\
q_{i} & - \text { velocity vector } \\
\boldsymbol{X} & - \text { external force } \\
x_{i} & - \text { the position vector } \\
\beta(=|d t / d z|) & - \text { uniform temperature gradient } \\
\beta^{\prime}(=|d c / d z|) & - \text { uniform solute gradient } \\
\Gamma_{i j} & - \text { stress tensor } \\
\delta_{i j} & - \text { Kroneckor delta } \\
\delta p & - \text { perturbation in pressure } \\
\delta \rho & - \text { perturbation in density } \\
\lambda & - \text { the stress relaxation time } \\
\lambda_{0} & - \text { the strain retardation time } \\
\mu & - \text { viscosity } \\
\mu_{e} & - \text { magnetic permeability } \\
\left(\mu / k_{1}\right) v & - \text { resistance term } \\
\rho & - \text { density } \\
\tau_{i j} & - \text { shearing stress tensor } \\
v & - \text { kinematic viscosity }
\end{aligned}
$$

\section{References}

Bhatia P.K. and Steiner J.M. (1972): Convective instability in a rotating viscoelastic fluid layer. - Z. Angew. Math. Mech., vol.52, pp.321.

Chandrasekhar S. (1981): Hydrodynamic and Hydromagnetic Stability. - New York: Dover Publications.

Gupta A.S. (1967): Hall effects on thermal instability. - Rev. Rumaine Math. Pure Appl., vol.12, pp.665.

Gupta U., Aggarwal P. and Wanchoo R.K. (2012): Thermal convection of dusty compressible Rivlin-Ericksen viscoelastic fluid with Hall currents. - Thermal Science, vol.16(1), p.191.

Ingham D.B. and Pop I. (1998): Transport phenomena in porous medium. - Oxford, UK: Pergamon Press.

Kumar P. and Singh G.J. (2006): Stability of two superposed Rivlin-Ericksen viscoelastic fluids in the presence of suspended particles. - Rom. J. Phys., vol.51, pp.927.

Kumar P. (2000): Rayleigh-Taylor instability of Rivlin-Ericksen elastico-viscous fluids in presence of suspended particles through porous medium. - Indian J. Pure Appl. Math., vol.31, p.533.

Kumar P., Lal R. and Sharma P. (2004): Instability of two rotating viscoelastic (Rivlin- Ericksen) superposed fluids with suspended particles in porous medium. - Rom. J. Phys., vol.49, p.209.

Kumar P., Lal R. and Singh M. (2007): Hydrodynamic and hydromagnetic stability of two stratified Rivlin-Ericksen elastico-viscous superposed fluid. - Int. J. Appl. Mech. Engng., vol.12, pp.645.

Kumar P., Singh G.J. and Roshan R. (2005): MHD instability of rotating superposed Rivlin-Ericksen viscoelastic fluids through porous medium. - Ganita Sandesh, Rajsthan Ganita Parishad, India, vol.19, p.89.

Lapwood E.R. (1948): Convection of a fluid in a porous medium. - Proc. Camb. Phil. Soc., vol.44, p.508.

Nield D.A. and Bejan A. (1999): Convection in Porous Medium (2 ${ }^{\text {nd }}$ edition). - New York, USA: Springer Verlag. 
Palaniswamy V.I. and Purushotham C.M. (1981): Stability of shear flow of stratified fluids with fine dust. - Phys. Fluids, vol.24, pp.1224.

Phillips O.M. (1991): Flow and Reaction in Permeable Rocks. - UK: Cambridge University Press.

Rivlin R.S. and Ericksen J.L. (1955): Stress-deformation relaxations for isotropic materials. - J. Rat. Mech. Anal., vol.4, pp.323.

Scanlon J.W. and Segel L.A. (1973): Some effects of suspended particles on the onset of Be'nard convection. - Phys. Fluids, vol.16, pp.1573.

Sharma R.C. (1976): Effect of rotation on thermal instability of a viscoelastic fluid. - Acta Physica Hung., vol.40, pp.11.

Sharma R.C. and Kumar P. (1996): Effect of rotation on thermal instability in Rivlin-Ericksen elastico-viscous fluid. Z. Naturforch., vol.51, p.821.

Sharma R.C. and Kumar P. (1997b): Hydrodynamic stability of Rivlin-Ericksen elastico-viscous superposed conducting fluids. - Z. Naturforch., vol.52a, p.528.

Sharma R.C. and Kumar P. (1997a): Thermal instability in Rivlin-Ericksen elastico-viscous fluid in hydromagnetics. Z. Naturforch., vol.52a, p.369.

Sherman A. and Sutton G.W. (1962): Magnetohydrodynamics. - Evanston, Illinois: Northwestern University Press.

Spiegel E.A. and Veronis G. (1960): On the Boussinesq approximation for a compressible fluid. - Astrophys. J., vol.131, pp.442.

Wooding R.A. (1960): Rayleigh instability of a thermal boundary layer in flow through a porous medium. - J. Fluid Mech., vol.9, p.183.

Received: September 7, 2012

Revised: May 29, 2013 